the biology of the organisms. Details of biochemistry, immunology and pathology are kept to a minimum, but enough information is given to inform the reader about various important facts without impeding the logical flow of the text. The style is buoyant and refreshing, and the book could be read from beginning to end with enjoyment. It is fully and reasonably well illustrated although not all the photographs are of the best quality. This is a volume that should be bought by all those working with haemoflagellates, their associated diseases or vectors, and by those taking postgraduate or even undergraduate courses in parasitology because it contains a vast amount of information not available in such an accessible form elsewhere.

There must, of course, be criticisms of a book of this kind and some readers, particularly those in South America, may feel that $T$. cruzi has been given a disproportionately small share of the text; be this as it may even those readers will learn something from the rest of the book. The references, however, are inadequate. There are less than 70 , grouped together at the end of the book without extensive cross-referencing between the text and the bibliography. Similarly, there is nothing to link the figures directly to their sources. These defects are likely to be particularly infuriating if the book is to be used as a bridge between textbook and research paper, but there will undoubtedly be further editions and such problems can be attended to then.

F.E.G. Cox is Professor of Zoology at King's College, University of London.

\section{All around reactors}

\section{Malcolm C. Scott}

Nuclear Fission Reactors.

By I.R. Cameron.

Plenum: 1983. Pp.390. \$42.50, £29.75.

THE nuclear industry is now well established, therefore requiring a supply of trained staff. Its growth is also contentious, making well-informed protagonists and antagonists essential if energy investment programmes are to be other than political slanging matches. Yet, despite these two factors, there have been relatively few books which have provided the fundamental introduction and the breadth required to suit both aspiring specialists and those wanting a more general background.

For this reason alone J.R. Cameron's Nuclear Fission Reactors must be welcomed. In addition, the author has a fluency of style and a clarity of presentation which makes the book a pleasure to read. Lest "pleasure" implies a lightweight work, let me hasten to add that part of the pleasure derives from seeing how the author manages to introduce a mass of detailed scientific and technical ideas, and interrelate them, and yet retain a lightness of touch which makes it all seem easy to understand.

Although the book stands as an integrated whole, giving a sound grounding in the physical and technological basis for nuclear power, Cameron has broken the subject down into four sections. The first, of three chapters, covers the physics of the atom and nucleus and then builds up, through a description of fission and the chain reaction, to an account of nuclear reactor theory, based on the diffusion approximation. Up to this point the ground is well trodden, though this is a particularly clear exposition, with worked examples to assist absorption.

The next three chapters are also general in scope. The first two cover the materials' aspects of nuclear fuel and other in-core materials. There are sections on fuel enrichment, and the importance of radiation damage is emphasized in the descriptions of individual materials. The next chapter, which concludes the general section, examines heat transfer and the steam cycle.

After a brief introduction, the third part of the book consists of four chapters, one on each of the main reactor types. Perhaps because of his background (one deduces that Cameron is an Englishman who has emigrated to Canada) the author has resisted the temptation to cover light-water reactors in detail to the exclusion of other systems. Instead we have roughly equal weight given to fast reactors, light-water, heavy-water and gas-cooled systems. Finally, a single chapter (the longest) considers safety and environmental effects, incorporating - inevitably and necessarily - a section on reactor accidents which includes an account of events at Three Mile Island. With characteristic thoroughness the author starts this chapter with several pages on the biological effects of radiation and finishes with a section on the long-term waste-disposal problem.

In a book of this breadth, individual specialists will find areas where emphases are felt to be misplaced or where information is, perhaps, a little dated. Additionally, a description of some of the reactor systems operating, or under consideration, in the USSR would have been interesting whilst some readers may be off put by the use of imperial units (surely an SI version will appear soon?). But these are minor quibbles. This is a roundly conceived and well-written book - still all too rare outside mainline undergraduate texts - which provides a superb introduction to nuclear reactors for the non-specialist and aspiring specialist alike.

Malcolm C. Scott is a Senior Lecturer in the Department of Physics at the University of Birmingham, and is in charge of the MSc course in the Physics and Technology of Nuclear Reactors.

\section{Stevenson's humus}

\author{
M.H.B. Hayes
}

Humus Chemistry: Genesis, Composition, Reactions.

By F.J. Stevenson.

Wiley: 1982. Pp.443. £37.95, \$53.15.

Humus, the product of biological and chemical transformations of plant and animal residues, is present in all soils and waters. In the soil environment humus substances stabilize soil aggregates, act as reservoirs for water and cations, and provide substrates for the teeming soil biota. After mineralization they yield valuable reserves of $\mathbf{N}, \mathbf{P}, \mathrm{S}$ and trace elements for plant growth. Less desirably, some humus components can inactivate soil-applied organic chemicals, and certain fragments of these components, with or without associated applied chemicals, could present health l.azards when transported into waterways.

Thirty years ago F.J. Stevenson made an outstanding contribution to our understanding of the electrokinetic properties of humic acids. Since that time he has been at the forefront of research in several other areas of humus chemistry, and there are few people better qualified to prepare a comprehensive treatise on the subject. His new book comes up to expectations almost fully.

Here Stevenson provides all of the pertinent quantitative background to humus materials, and he discusses their environmental significance in detail. His treatment of the genesis of humus is, in my view, the best available. Similarly his coverage of extraction and fractionation procedures, and the treatment of soil saccharides, organic nitrogen, phosphorus, sulphur and lipids in humus is clear and to the point. Interpretations of studies of the surface and colloidal properties of the polymers, and of their binding of heavy metals are outstanding.

In view of the importance of humus materials, both in soils and water, it is perhaps surprising that so little is known of their structures. Stevenson rightly points out that a better knowledge of their formation could help overcome this deficiency. The difficulty lies in the intractable yet intriguing chemical make-up of the polymers in which the labile peptide bonds and glycosidic linkages are peripheral to the core structures. These structures are held together by difficult to cleave carboncarbon bonds and ether linkages, and so it is hard to degrade humic materials to products which can be related to primary structures. Because their synthesis is not genetically controlled, it is possible that very few molecules are similar in any batch, and hence attempts at determining secondary structures would be meaningless. The information which is available about tertiary structures is considerably better, 